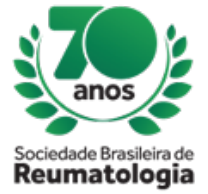

\title{
MCARDLE'S DISEASE TRIGGERED AFTER VACCINATION AGAINST YELLOW FEVER: CASE REPORT
}

Dayrana Alves Lucena (HSPE - IAMSPE, São Paulo, SP, Brasil), Danielle Arraes Rubini (HSPE - IAMSPE, São Paulo, SP, Brasil), Marcelo Nora Resende (HSPE - IAMSPE, São Paulo, SP, Brasil), Tamires Guimarães Costa (HSPE - IAMSPE, São Paulo, SP, Brasil), Renata Ferreira Rosa (HSPE - IAMSPE, São Paulo, SP, Brasil), Nafice Costa Araújo (HSPE - IAMSPE, São Paulo, SP, Brasil), Rina Dalva Neubath Giorgi (HSPE - IAMSPE, São Paulo, SP, Brasil)

\section{BACKGROUND}

In the last decade, yellow fever has presented an epizootic-epidemic pattern, manifesting itself as a reemerging disease in new areas of the Brazilian territory. The yellow fever vaccine (YFV) is composed of live attenuated virus and is highly immunogenic. Adverse events associated with YFV may occur as local and systemic reactions, the latter ranging from moderate to severe, including death. Fever, headache and myalgia have been the most frequently reported events. We will report the case of a patient who presented severe adverse effect after YFV and triggered a metabolic myopathy.

\section{CASE REPORT}

VJS, 34 years old, male, previously healthy, presented after five days of vaccination against yellow fever with coluria, abdominal pain, dyspnea on medium exertion, pain and muscle weakness. He sought Hospital de Pirituba, where he was hospitalized for myopathy, liver disease and renal insufficiency requiring dialysis therapy. After improving renal function, the patient maintained high CPK values, which is why he was referred to our Hospital. Initially it was evaluated in the infectology and in the neurology, being sent to our Service with FAN 1/640 thick dotted nuclear pattern and creatine phosphokinase of $1945 \mathrm{IU} / \mathrm{L}$. The autoantibody profile and upper and lower limb electromyography were normal. Muscle biopsy evidenced a vacuolar myopathic pattern, suggestive of glycogenosis, deficient in the labeling for myophosphorylase (Glycogenosis type V - McArdle's disease).

\section{CONCLUSION}

Severe adverse events associated with AFV include hypersensitivity reactions, acute neurological disease (encephalitis, meningitis, autoimmune diseases involving central and peripheral nervous system involvement), and acute viscerotropic disease (hepatitis, renal failure and haemorrhages). Our patient evolved with a severe adverse effect and triggered a primary metabolic myopathy. McArdle's Disease is the most common muscular glycogenosis, whose symptoms usually manifest in childhood and adolescence after physical activity. Some patients may manifest symptoms in adulthood with proximal weakness and cramps associated with a degree of rhabdomyolysis after brief, intense episodes of exercise. This case denotes the importance of differential diagnosis in the various myopathies, be it associated with drugs, infectious processes, vaccines, autoimmune and metabolic inflammatory myopathies, emphasizing the need for muscle biopsy. 